PROCEEDINGS OF THE

AMERICAN MATHEMATICAL SOCIETY

Volume 137, Number 8, August 2009, Pages 2581-2590

S 0002-9939(09)09814-1

Article electronically published on February 17, 2009

\title{
A COMBINATORIAL INTERPRETATION OF THE LEGENDRE-STIRLING NUMBERS
}

\author{
GEORGE E. ANDREWS AND LANCE L. LITTLEJOHN
}

(Communicated by Jim Haglund)

\begin{abstract}
The Legendre-Stirling numbers were discovered in 2002 as a result of a problem involving the spectral theory of powers of the classical secondorder Legendre differential expression. Specifically, these numbers are the coefficients of integral composite powers of the Legendre expression in Lagrangian symmetric form. Quite remarkably, they share many similar properties with the classical Stirling numbers of the second kind which, as shown by Littlejohn and Wellman, are the coefficients of integral powers of the Laguerre differential expression. An open question regarding the Legendre-Stirling numbers has been to obtain a combinatorial interpretation of these numbers. In this paper, we provide such an interpretation.
\end{abstract}

\section{INTRODUCTION}

The Legendre-Stirling numbers $\left\{P S_{n}^{(j)}\right\}$ were first discovered (see [7) as a result of an application of left-definite operator theory to the classical second-order Legendre differential equation

$$
\ell_{k}[y](x):=-\left(\left(1-x^{2}\right) y^{\prime}(x)\right)^{\prime}+k y(x)=\lambda y(x) \quad(x \in(-1,1)) .
$$

Here, $k$ is a fixed, non-negative constant. In most special functions settings, we take $k=0$; for operator-theoretic purposes, it is sometimes useful to have $k>0$ and we can do so, without loss of generality, since we can negate the additional term $k y$ by also including it as part of the spectral term $\lambda y$. The classical (first) left-definite setting for this expression is in the Sobolev space

$$
H=\left\{f:(-1,1) \rightarrow \mathbb{C} \mid f \in A C_{\text {loc }}(-1,1), f, \sqrt{1-x^{2}} f^{\prime} \in L^{2}(-1,1)\right\},
$$

with inner product

$$
(f, g)=\int_{-1}^{1}\left(\left(1-x^{2}\right) f^{\prime}(x) \bar{g}^{\prime}(x)+k f(x) \bar{g}(x)\right) d x \quad(f, g \in H) ;
$$

notice that this inner product (when $k>0$ ) is generated from the left-hand side of (1.1), prompting the notation 'left-definite'. Left-definite theory has its origins in differential equations and can be traced back to some fundamental work of Weyl

Received by the editors September 2, 2008, and, in revised form, October 21, 2008.

2000 Mathematics Subject Classification. Primary 05A05, 05A15, 33C45; Secondary 34B24, 34L05, 47E05.

Key words and phrases. Legendre-Stirling numbers, Stirling numbers of the second kind, Legendre polynomials, left-definite theory, self-adjoint operator.

(C)2009 American Mathematical Society Reverts to public domain 28 years from publication 
in 13. Questions about the existence of symmetric or self-adjoint operators, generated by (1.1), in $H$ and about their spectral properties are the central issues in a left-definite analysis of the Legendre differential expression. This left-definite study of (1.1) in $H$ was initiated by Pleijel in a series of papers [11] and [12] and further studied by Everitt in [5. Specifically, these authors sought a self-adjoint operator in $H$, generated by (1.1), that has the Legendre polynomials $\left\{P_{m}\right\}_{m=0}^{\infty}$ as (orthogonal) eigenfunctions in $H$.

In [9, Littlejohn and Wellman generalized left-definite theory from its traditional roots in differential equations to a more abstract setting, namely to arbitrary selfadjoint operators $A$ that are bounded below in a Hilbert space $(H,(\cdot, \cdot))$ by a positive constant $k$; that is to say,

$$
(A x, x) \geq k(x, x) \quad(x \in \mathcal{D}(A)) .
$$

They show that the appropriate (first) left-definite setting for such operators is the Hilbert space $H_{1}=\mathcal{D}\left(A^{1 / 2}\right)$ endowed with the inner product

$$
(f, g)_{1}=\left(A^{1 / 2} f, A^{1 / 2} g\right) \quad\left(f, g \in H_{1}\right) .
$$

Moreover, using the Hilbert space spectral theorem, they prove that there is, in fact, a continuum of left-definite spaces $\left\{\left(H_{r},(\cdot, \cdot)_{r}\right)\right\}_{r>0}$ associated with the pair $(H, A)$; these spaces are explicitly given by

$$
H_{r}=\mathcal{D}\left(A^{r / 2}\right)
$$

with corresponding inner products

$$
(f, g)_{r}=\left(A^{r / 2} f, A^{r / 2} g\right) \quad\left(f, g \in H_{r}\right) .
$$

In practice, unless the spectral resolution of the identity is explicitly known for the self-adjoint operator $A$, it is difficult to determine $H_{r}$ for every $r>0$. However, as the authors show in [9] (see also [3], 6], 7], and [8]), it is possible to compute these spaces and inner products for several well-known self-adjoint operators for each positive integer $r$. Indeed, for $f \in \mathcal{D}\left(A^{r}\right) \subset \mathcal{D}\left(A^{r / 2}\right)$ and $g \in \mathcal{D}\left(A^{r / 2}\right)$, the self-adjointness of $A^{r / 2}$ yields

$$
(f, g)_{r}=\left(A^{r} f, g\right) .
$$

Consequently, the $r^{\text {th }}$ left-definite inner product, as well as the $r^{\text {th }}$ left-definite space $H_{r}$, is generated by the $r^{t h}$ power of $A$. In particular, when $A$ is generated by a differential expression $\ell[\cdot]$ such as (1.1), this means that knowing the explicit form of the $r^{t h}$ power of $\ell[\cdot]$ is necessary in order to find both the $r^{t h}$ left-definite space and the $r^{t h}$ left-definite inner product. It is precisely these integral powers of the Legendre expression that involve the Legendre-Stirling numbers.

The contents of this paper are as follows. In Section 2, we discuss the integral powers of the Legendre expression $\ell[\cdot]$ and briefly introduce the Legendre-Stirling numbers from the context of left-definite theory. As a means of comparison, we also discuss the classical Stirling numbers of the second kind in Section 2 these combinatorial numbers are well known and we discuss a relatively new result involving these numbers and the powers of the classical second-order Laguerre differential expression. In Section 3, we compare various properties of the classical Stirling numbers of the section kind with the Legendre-Stirling numbers; as we will see, these two sets of numbers are similar in many ways. One such property, the triangular recurrence relation (TRR), is paramount in establishing a combinatorial interpretation of the Legendre-Stirling numbers, so we prove the TRR for the Legendre-Stirling 
numbers in Section 3. In Section 4, we derive our combinatorial interpretation of the Legendre-Stirling numbers and illustrate this result with several examples.

\section{BACKGROUND}

In [7, the authors prove the following result, which is the key prerequisite to establishing the left-definite theory of the Legendre differential expression; it is in this result that the Legendre-Stirling numbers are first introduced.

Theorem 1. Let $n \in \mathbb{N}$. The $n^{\text {th }}$ composite power of the Legendre differential expression (1.1), in Lagrangian symmetric form, is given by

$$
\ell_{k}^{n}[y](x)=\sum_{j=0}^{n}(-1)^{j}\left(a_{j}(n, k)\left(1-x^{2}\right)^{(j)} y^{(j)}(x)\right)^{(j)} \quad(x \in(-1,1)),
$$

where the coefficients $a_{j}(n, k)(j=0,1, \ldots, n)$ are non-negative and given by

$$
a_{0}(n, k)= \begin{cases}0 & \text { if } k=0 \\ k^{n} & \text { if } k>0\end{cases}
$$

and

$$
a_{j}(n, k):=\left\{\begin{array}{ll}
P S_{n}^{(j)} & \text { if } k=0 \\
\sum_{r=0}^{n-j}\left(\begin{array}{l}
n \\
r
\end{array}\right) P S_{n-r}^{(j)} k^{r} & \text { if } k>0
\end{array} \quad(j \in\{1, \ldots, n\}) ;\right.
$$

moreover, for $n, j \in \mathbb{N}$, each $P S_{n}^{(j)}$ is positive and given by

$$
P S_{n}^{(j)}=\sum_{m=1}^{j}(-1)^{m+j} \frac{(2 m+1)\left(m^{2}+m\right)^{n}}{(m+j+1) !(j-m) !} .
$$

In particular,

$$
\ell_{0}^{n}[y](x)=\sum_{j=1}^{n}(-1)^{j}\left(P S_{n}^{(j)}\left(1-x^{2}\right)^{(j)} y^{(j)}(x)\right)^{(j)} \quad(x \in(-1,1)) .
$$

Furthermore, $P S_{n}^{(j)}$ is the coefficient of $x^{n-j}$ in the Taylor series expansion of

$$
f_{j}(x)=\prod_{m=0}^{j} \frac{1}{1-m(m+1) x} \quad\left(|x|<\frac{1}{j(j+1)}\right) .
$$

We call the numbers $\left\{P S_{n}^{(j)}\right\}$ the Legendre-Stirling numbers. From (2.3), we see that we can extend the definition of these numbers to include the initial conditions

$$
P S_{n}^{(0)}=0 \text { and } P S_{0}^{(j)}=0 \text { except } P S_{0}^{(0)}=1 \quad(n, j \in \mathbb{N}) .
$$

The focus of this paper is on the combinatorics of these Legendre-Stirling numbers; however, for the sake of completeness, we note that the $n^{\text {th }}$ left-definite inner product and space are readily obtained from Theorem 1. Indeed, from (2.1), it can be shown that the $n^{\text {th }}$ left-definite inner product is given by

$$
(f, g)_{n}:=\sum_{j=0}^{n} \int_{-1}^{1} a_{j}(n, k)\left(1-x^{2}\right)^{j} f^{(j)}(x) \bar{g}^{(j)}(x) d x .
$$

Moreover, as shown in [7, with the $m^{\text {th }}$ Legendre polynomial defined by

$$
P_{m}(x)=\sqrt{\frac{2 m+1}{2}} \sum_{j=0}^{[m / 2]} \frac{(-1)^{j}(2 m-2 j) !}{2^{m} j !(m-j) !(m-2 j) !} x^{m-2 j} \quad\left(m \in \mathbb{N}_{0}\right),
$$


the Legendre polynomials $\left\{P_{m}\right\}_{m=0}^{\infty}$ satisfy the orthogonality relationship

$$
\left(P_{m}, P_{r}\right)_{n}=(m(m+1)+k)^{n} \delta_{m, r} \quad\left(m, r \in \mathbb{N}_{0}\right)
$$

and form a complete orthogonal set in the $n^{\text {th }}$ left-definite space explicitly given by

$$
\begin{aligned}
H_{n}=\{f:(-1,1) \rightarrow \mathbb{C} \mid & f, f^{\prime}, \ldots, f^{(n-1)} \in A C_{\mathrm{loc}}(-1,1) ; \\
& \left.\left(1-x^{2}\right)^{j / 2} f^{(j)} \in L^{2}(-1,1)(j=0,1, \ldots n)\right\} .
\end{aligned}
$$

The Legendre-Stirling numbers have several properties similar to the classical Stirling numbers of the second kind $\left\{S_{n}^{(j)}\right\}$ (see [1, pp. 824-825] and [4, Chapter V]). Indeed, in a new application of the Stirling numbers of the second kind, it is reported in [9] that the Stirling numbers of the second kind $\left\{S_{n}^{(j)}\right\}$ are the coefficients of the integral composite powers of the second-order Laguerre differential expression:

$$
m_{k}[y](x):=\frac{1}{x^{\alpha} e^{-x}}\left(-\left(x^{\alpha+1} e^{-x} y^{\prime}(x)\right)^{\prime}+k x^{\alpha} e^{-x} y(x)\right) \quad(x \in(0, \infty)) .
$$

Specifically, the authors in 9 show that for each $n \in \mathbb{N}$,

$$
m_{k}^{n}[y](x)=\frac{1}{x^{\alpha} e^{-x}} \sum_{j=1}^{n}(-1)^{j}\left(b_{j}(n, k) x^{\alpha+j} e^{-x} y^{(j)}(x)\right)^{(j)},
$$

where

$$
b_{0}(n, k)= \begin{cases}0 & \text { if } k=0 \\ k^{n} & \text { if } k>0\end{cases}
$$

and

$$
b_{j}(n, k)=\left\{\begin{array}{ll}
S_{n}^{(j)} & \text { if } k=0 \\
\sum_{r=0}^{n-1}\left(\begin{array}{l}
n \\
r
\end{array}\right) S_{n-r}^{(j)} k^{r} & \text { if } k>0
\end{array} \quad(j=1,2, \ldots, n) .\right.
$$

In particular,

$$
m_{0}^{n}[y](x)=\frac{1}{x^{\alpha} e^{-x}} \sum_{j=1}^{n}(-1)^{j}\left(S_{n}^{(j)} x^{\alpha+j} e^{-x} y^{(j)}(x)\right)^{(j)} .
$$

We note that the Stirling numbers of the second kind also appear in the composite integral powers of the classical second-order Hermite differential equation

$$
h[y](x):=\frac{1}{\exp \left(-x^{2}\right)}\left(-\left(\exp \left(-x^{2}\right) y^{\prime}(x)\right)^{\prime}+k \exp \left(-x^{2}\right) y(x)\right) ;
$$

see [6] for further details.

\section{A comparison of Stirling numbers of the SeCOnd Kind AND THE LEGENDRE-STIRLING NUMBERS}

We begin this section with Table 1, which compares various properties of the Stirling numbers of the second kind and the Legendre-Stirling numbers; details and proofs of these properties are forthcoming in 2.

From this table, notice the rational generating functions for the Stirling numbers of the second kind and for the Legendre-Stirling numbers; in particular, note the coefficients $r$ and $r(r+1)$ in the denominators of these products. Remarkably, and perhaps somewhat mysteriously, these coefficients are, respectively, the eigenvalues that produce the Laguerre and Legendre polynomial solutions of degree $r$ in the Laguerre and Legendre differential equations. We find it even more remarkable that the computation of the integral composite powers of both the Laguerre and 
Table 1. A Comparison of Properties of Stirling Numbers of the Second Kind and Legendre-Stirling Numbers

\begin{tabular}{|l|l|l|}
\hline Property & Stirling Numbers 2nd Kind & Legendre-Stirling Numbers \\
\hline Vertical RR & $S_{n}^{(j)}=\sum_{r=j}^{n} S_{r-1}^{(j-1)} j^{n-r}$ & $P S_{n}^{(j)}=\sum_{r=j}^{n} P S_{r-1}^{(j-1)}(j(j+1))^{n-r}$ \\
\hline Rational GF & $\prod_{r=1}^{j} \frac{1}{1-r x}=\sum_{n=0}^{\infty} S_{n}^{(j)} x^{n-j}$ & $\prod_{r=0}^{j} \frac{1}{1-r(r+1) x}=\sum_{n=0}^{\infty} P S_{n}^{(j)} x^{n-j}$ \\
\hline Triangular RR & $S_{n}^{(j)}=S_{n-1}^{(j-1)}+j S_{n-1}^{(j)}$ & $P S_{n}^{(j)}=P S_{n-1}^{(j-1)}+j(j+1) P S_{n-1}^{(j)}$ \\
\hline & $S_{n}^{(0)}=S_{0}^{(j)}=0 ; S_{0}^{(0)}=1$ & $P S_{n}^{(0)}=P S_{0}^{(j)}=0 ; P S_{0}^{(0)}=1$ \\
\hline Horizontal GF & $x^{n}=\sum_{j=0}^{n} S_{n}^{(j)}(x)_{j}$ where & $x^{n}=\sum_{j=0}^{n} P S_{n}^{(j)}\langle x\rangle_{j}$ where \\
\hline & $(x)_{j}=x(x-1) \ldots(x-j+1)$ & $\langle x\rangle_{j}=x(x-2) \ldots(x-(j-1) j)$ \\
\hline 1st Kind Numbers & $(x)_{n}=\sum_{j=0}^{n} s_{n}^{(j)} x^{j}$ & $\langle x\rangle_{n}=\sum_{j=0}^{n} p s_{n}^{(j)} x^{j}$ \\
\hline
\end{tabular}

Legendre differential equations (these are completely algebraic calculations) are therefore intimately connected to these classical orthogonal polynomial solutions. Furthermore, in the case of the Legendre expression, the Glazman-Krein-Naimark theory [10] implies that there is an uncountable number of self-adjoint operators in $L^{2}(-1,1)$, generated by the Legendre expression $\ell_{0}[\cdot]$, each of which has a purely discrete (that is, eigenvalues only) spectrum. Exactly one of these self-adjoint operators, namely the 'Legendre polynomial' operator $A$ defined by

$$
\begin{aligned}
& A f(x)=\ell_{0}[f](x) \quad(\text { a.e. } x \in(-1,1)), \\
& \mathcal{D}(A)=\left\{f:(-1,1) \rightarrow \mathbb{C} \mid f, f^{\prime} \in A C_{\mathrm{loc}}(-1,1) ; f, \ell_{0}[f] \in L^{2}(-1,1) ;\right. \\
&\left.\lim _{x \rightarrow \pm 1}\left(1-x^{2}\right) f^{\prime}(x)=0\right\}
\end{aligned}
$$

has spectrum $\left\{r(r+1) \mid r \in \mathbb{N}_{0}\right\}$. Why does the horizontal generating function involve the eigenvalues $r(r+1)$ of this operator $A$ ? Why doesn't this generating function involve the eigenvalues of one of the other self-adjoint operators? It seems that there is an interesting connection here that deserves further attention.

Tables 2 and 3 list several Legendre-Stirling numbers and, for comparison purposes, Stirling numbers of the second kind.

TABle 2. A List of Legendre-Stirling Numbers (for example, $\left.P S_{4}^{(3)}=20, P S_{6}^{(4)}=1092\right)$

\begin{tabular}{|l||l|l|l|l|l|l|l|l|l|l|}
\hline$j / n$ & $n=1$ & $n=2$ & $n=3$ & $n=4$ & $n=5$ & $n=6$ & $n=7$ & $n=8$ & $n=9$ & $n=10$ \\
\hline \hline$j=1$ & 1 & 2 & 4 & 8 & 16 & 32 & 64 & 128 & 256 & 512 \\
\hline$j=2$ & - & 1 & 8 & 52 & 320 & 1936 & 11648 & 69952 & 419840 & 2519296 \\
\hline$j=3$ & - & - & 1 & 20 & 292 & 3824 & 47824 & 585536 & 7096384 & 85576448 \\
\hline$j=4$ & - & - & - & 1 & 40 & 1092 & 25664 & 561104 & 11807616 & 243248704 \\
\hline$j=5$ & - & - & - & - & 1 & 70 & 3192 & 121424 & 4203824 & 137922336 \\
\hline$j=6$ & - & - & - & - & - & 1 & 112 & 7896 & 453056 & 23232176 \\
\hline$j=7$ & - & - & - & - & - & - & 1 & 168 & 17304 & 1422080 \\
\hline$j=8$ & - & - & - & - & - & - & - & 1 & 240 & 34584 \\
\hline$j=9$ & - & - & - & - & - & - & - & - & 1 & 330 \\
\hline$j=10$ & - & - & - & - & - & - & - & - & - & 1 \\
\hline
\end{tabular}


TABLe 3. A List of Stirling Numbers of the Second Kind $S_{n}^{(j)}$ (for example, $S_{4}^{(2)}=7, S_{6}^{(4)}=65$ )

\begin{tabular}{|l||l|l|l|l|l|l|l|l|l|l|}
\hline$j / n$ & $n=1$ & $n=2$ & $n=3$ & $n=4$ & $n=5$ & $n=6$ & $n=7$ & $n=8$ & $n=9$ & $n=10$ \\
\hline \hline$j=1$ & 1 & 1 & 1 & 1 & 1 & 1 & 1 & 1 & 1 & 1 \\
\hline$j=2$ & - & 1 & 3 & 7 & 15 & 31 & 63 & 127 & 255 & 511 \\
\hline$j=3$ & - & - & 1 & 6 & 25 & 90 & 301 & 966 & 3025 & 9330 \\
\hline$j=4$ & - & - & - & 1 & 10 & 65 & 350 & 1701 & 7770 & 34105 \\
\hline$j=5$ & - & - & - & - & 1 & 15 & 140 & 1050 & 6951 & 42525 \\
\hline$j=6$ & - & - & - & - & - & 1 & 21 & 266 & 2646 & 22827 \\
\hline$j=7$ & - & - & - & - & - & - & 1 & 28 & 462 & 5880 \\
\hline$j=8$ & - & - & - & - & - & - & - & 1 & 36 & 750 \\
\hline$j=9$ & - & - & - & - & - & - & - & - & 1 & 45 \\
\hline$j=10$ & - & - & - & - & - & - & - & - & - & 1 \\
\hline
\end{tabular}

The next lemma establishes the triangular recurrence relation for the LegendreStirling numbers; we need this result for our main theorem in the next section.

Lemma 3.1. The Legendre-Stirling numbers satisfy the following triangular recurrence relation:

$$
\begin{aligned}
& P S_{n}^{(j)}=P S_{n-1}^{(j-1)}+j(j+1) P S_{n-1}^{(j)} \quad(n, j \in \mathbb{N}, j \leq n), \\
& P S_{n}^{(0)}=P S_{0}^{(j)}=0 ; P S_{0}^{(0)}=1 \quad(n, j \in \mathbb{N}) .
\end{aligned}
$$

Proof. The initial conditions given above are part of the definition of $P S_{n}^{(j)}$, given in (2.4). On the other hand, for $n, j \in \mathbb{N}$ and $j \leq n$,

$$
\begin{aligned}
& P S_{n-1}^{(j-1)}+j(j+1) P S_{n-1}^{(j)} \\
& =\sum_{r=1}^{j-1}(-1)^{r+j-1} \frac{(2 r+1)\left(r^{2}+r\right)^{n-1}}{(r+j) !(j-1-r) !}+j(j+1) \sum_{r=1}^{j}(-1)^{r+j} \frac{(2 r+1)\left(r^{2}+r\right)^{n-1}}{(r+j+1) !(j-r) !} .
\end{aligned}
$$

A routine calculation shows that, for $1 \leq r \leq j-1$,

$$
\begin{aligned}
& (-1)^{r+j-1} \frac{(2 r+1)\left(r^{2}+r\right)^{n-1}}{(r+j) !(j-1-r) !}+j(j+1)(-1)^{r+j} \frac{(2 r+1)\left(r^{2}+r\right)^{n-1}}{(r+j+1) !(j-r) !} \\
& =(-1)^{r+j} \frac{(2 r+1)\left(r^{2}+r\right)^{n}}{(r+j+1) !(j-r) !} .
\end{aligned}
$$

Moreover, the term corresponding to $r=j$ in the second sum in (3.3) is

$$
j(j+1)(-1)^{2 j} \frac{(2 j+1)\left(j^{2}+j\right)^{n-1}}{(2 j+1) !}=\frac{(2 j+1)\left(j^{2}+j\right)^{n}}{(2 j+1) !},
$$

which is the same as the term in (3.4) when $r=j$. Consequently, the right-hand side of (3.3) simplifies to

$$
\sum_{r=1}^{j}(-1)^{r+j} \frac{(2 r+1)\left(r^{2}+r\right)^{n}}{(r+j+1) !(j-r) !}=P S_{n}^{(j)} .
$$




\section{A COMbinatorial interpretation OF THE LEGENDRE-STIRLING NUMBERS}

The Stirling number of the second kind $S_{n}^{(j)}$ counts the number of ways of putting $n$ objects into $j$ non-empty, indistinguishable sets. For an excellent account of these numbers, and a full account of their properties, see the text of Comtet [4, Chapter V]. From Tables 2 and 3 in the previous section, it is immediately clear that, for $n>j, P S_{n}^{(j)}$ is considerably larger than $S_{n}^{(j)}$. It is natural to ask: what do the Legendre-Stirling numbers count? In this section, we answer this question.

To describe a combinatorial interpretation of the Legendre-Stirling number $P S_{n}^{(j)}$, for each $n \in \mathbb{N}$, we consider two copies of each positive integer between 1 and $n$ :

$$
1_{1}, 1_{2}, 2_{1}, 2_{2}, \ldots, n_{1}, n_{2}
$$

we may say that these are the integers $\{1,2, \ldots, n\}$ with two colors. For positive integers $p, q \leq n$ and $i, j \in\{1,2\}$, we say that $p_{i}>q_{j}$ if $p>q$. We now describe two rules on how to fill $j+1$ 'boxes' with the numbers $\left\{1_{1}, 1_{2}, 2_{1}, 2_{2}, \ldots, n_{1}, n_{2}\right\}$ :

(1) The 'zero box' is the only box that may be empty and it may not contain both copies of any number.

(2) The other $j$ boxes are indistinguishable and each is non-empty; for each such box, the smallest element in that box must contain both copies (or colors) of this smallest number but no other elements have both copies in that box.

Example 4.1. $n=3, j=2$. As Table 4 shows, there are eight different ways to satisfy the above two rules.

TABLE 4

\begin{tabular}{|l|l|l|l|}
\hline Zero box & Other two boxes & Zero box & Other two boxes \\
\hline$\varnothing$ & $\left\{1_{1}, 1_{2}, 3_{1}\right\},\left\{2_{1}, 2_{2}, 3_{2}\right\}$ & $\left\{3_{1}\right\}$ & $\left\{1_{1}, 1_{2}, 3_{2}\right\},\left\{2_{1}, 2_{2}\right\}$ \\
\hline$\varnothing$ & $\left\{1_{1}, 1_{2}, 3_{2}\right\},\left\{2_{1}, 2_{2}, 3_{1}\right\}$ & $\left\{3_{2}\right\}$ & $\left\{1_{1}, 1_{2}, 3_{1}\right\},\left\{2_{1}, 2_{2}\right\}$ \\
\hline$\left\{2_{1}\right\}$ & $\left\{1_{1}, 1_{2}, 2_{2}\right\},\left\{3_{1}, 3_{2}\right\}$ & $\left\{3_{1}\right\}$ & $\left\{1_{1}, 1_{2}\right\},\left\{2_{1}, 2_{2}, 3_{2}\right\}$ \\
\hline$\left\{2_{2}\right\}$ & $\left\{1_{1}, 1_{2}, 2_{1}\right\},\left\{3_{1}, 3_{2}\right\}$ & $\left\{3_{2}\right\}$ & $\left\{1_{1}, 1_{2}\right\},\left\{2_{1}, 2_{2}, 3_{1}\right\}$ \\
\hline
\end{tabular}

Example 4.2. $n=5, j=1$. In this case, there are sixteen different ways to satisfy the above two rules. To see this, first note that no copy of the number 1 can be in the zero box. Indeed, by Rule 1, it is not possible for both copies of 1 to belong to the zero box. Moreover, if the zero box contains exactly one copy of 1 , then the only other box in this case must contain the other copy of 1 , which violates Rule 2 . In fact, since there is only one other box besides the zero box, it is straightforward to see that the zero box is non-empty and necessarily has the form $\left\{2_{i}, 3_{j}, 4_{k}, 5_{l}\right\}$ where $i, j, k, l \in\{1,2\}$. There are two different choices for each of the numbers $2,3,4$, and 5 , and, consequently, there are precisely $2^{4}=16$ ways to build this zero box.

Example 4.3. $n=4, j=3$. For this example, there are twenty different possibilities. See Table 5.

We now come to the main result of this paper. 
TABLE 5

\begin{tabular}{|l|l|l|l|}
\hline $\begin{array}{l}\text { Zero } \\
\text { box }\end{array}$ & Other three boxes & $\begin{array}{l}\text { Zero } \\
\text { box }\end{array}$ & Other three boxes \\
\hline$\varnothing$ & $\left\{1_{1}, 1_{2}, 3_{1}\right\},\left\{2_{1}, 2_{2}, 3_{2}\right\},\left\{4_{1}, 4_{2}\right\}$ & $\left\{3_{1}\right\}$ & $\left\{1_{1}, 1_{2}, 3_{2}\right\},\left\{2_{1}, 2_{2}\right\},\left\{4_{1}, 4_{2}\right\}$ \\
\hline$\varnothing$ & $\left\{1_{1}, 1_{2}, 3_{2}\right\},\left\{2_{1}, 2_{2}, 3_{1}\right\},\left\{4_{1}, 4_{2}\right\}$ & $\left\{3_{2}\right\}$ & $\left\{1_{1}, 1_{2}, 3_{1}\right\},\left\{2_{1}, 2_{2}\right\},\left\{4_{1}, 4_{2}\right\}$ \\
\hline$\varnothing$ & $\left\{1_{1}, 1_{2}, 4_{1}\right\},\left\{2_{1}, 2_{2}, 4_{2}\right\},\left\{3_{1}, 3_{2}\right\}$ & $\left\{3_{1}\right\}$ & $\left\{1_{1}, 1_{2}\right\},\left\{2_{1}, 2_{2}, 3_{2}\right\},\left\{4_{1}, 4_{2}\right\}$ \\
\hline$\varnothing$ & $\left\{1_{1}, 1_{2}, 4_{2}\right\},\left\{2_{1}, 2_{2}, 4_{1}\right\},\left\{3_{1}, 3_{2}\right\}$ & $\left\{3_{2}\right\}$ & $\left\{1_{1}, 1_{2}\right\},\left\{2_{1}, 2_{2}, 3_{1}\right\},\left\{4_{1}, 4_{2}\right\}$ \\
\hline$\varnothing$ & $\left\{1_{1}, 1_{2}, 4_{1}\right\},\left\{2_{1}, 2_{2}\right\},\left\{3_{1}, 3_{2}, 4_{2}\right\}$ & $\left\{4_{1}\right\}$ & $\left\{1_{1}, 1_{2}\right\},\left\{2_{1}, 2_{2}, 4_{2}\right\},\left\{3_{1}, 3_{2}\right\}$ \\
\hline$\varnothing$ & $\left\{1_{1}, 1_{2}, 4_{2}\right\},\left\{2_{1}, 2_{2}\right\},\left\{3_{1}, 3_{2}, 4_{1}\right\}$ & $\left\{4_{2}\right\}$ & $\left\{1_{1}, 1_{2}\right\},\left\{2_{1}, 2_{2}, 4_{1}\right\},\left\{3_{1}, 3_{2}\right\}$ \\
\hline$\varnothing$ & $\left\{1_{1}, 1_{2}\right\},\left\{2_{1}, 2_{2}, 4_{1}\right\},\left\{3_{1}, 3_{2}, 4_{2}\right\}$ & $\left\{4_{1}\right\}$ & $\left\{1_{1}, 1_{2}\right\},\left\{2_{1}, 2_{2}\right\},\left\{3_{1}, 3_{2}, 4_{2}\right\}$ \\
\hline$\varnothing$ & $\left\{1_{1}, 1_{2}\right\},\left\{2_{1}, 2_{2}, 4_{2}\right\},\left\{3_{1}, 3_{2}, 4_{1}\right\}$ & $\left\{4_{2}\right\}$ & $\left\{1_{1}, 1_{2}\right\},\left\{2_{1}, 2_{2}\right\},\left\{3_{1}, 3_{2}, 4_{1}\right\}$ \\
\hline$\left\{2_{1}\right\}$ & $\left\{1_{1}, 1_{2}, 2_{2}\right\},\left\{3_{1}, 3_{2}\right\},\left\{4_{1}, 4_{2}\right\}$ & $\left\{4_{1}\right\}$ & $\left\{1_{1}, 1_{2}, 4_{2}\right\},\left\{2_{1}, 2_{2}\right\},\left\{3_{1}, 3_{2}\right\}$ \\
\hline$\left\{2_{2}\right\}$ & $\left\{1_{1}, 1_{2}, 2_{1}\right\},\left\{3_{1}, 3_{2}\right\},\left\{4_{1}, 4_{2}\right\}$ & $\left\{4_{2}\right\}$ & $\left\{1_{1}, 1_{2}, 4_{1}\right\},\left\{2_{1}, 2_{2}\right\},\left\{3_{1}, 3_{2}\right\}$ \\
\hline
\end{tabular}

Theorem 2. For $n, j \in \mathbb{N}_{0}$ and $j \leq n$, the Legendre-Stirling number $P S_{n}^{(j)}$ is the number of different distributions according to the above two rules.

Proof. Let $L e_{n}^{(j)}$ denote the number of different ways of distributing the numbers

$$
1_{1}, 1_{2}, 2_{1}, 2_{2}, \ldots, n_{1}, n_{2}
$$

into the "zero box" and the other $j$ indistinguishable boxes according to the above rules. Clearly $L e_{0}^{(0)}=1$ since there is only the zero box in this case and it is empty. Also $L e_{n}^{(0)}=0$ for $n \in \mathbb{N}$ since the rules stipulate that you cannot put $i_{1}$ and $i_{2}$ into the zero box, the only box available in this case. Next, $L e_{0}^{(j)}=0$ for $j>0$ since none of the $j$ indistinguishable boxes can be empty. Thus the initial conditions for $L e_{n}^{(j)}$ agree with those of $P S_{n}^{(j)}$ (see (3.2) ). Now we must establish the recurrence relation (3.1); to do so, we split the distributions into two exhaustive, disjoint sets:

(I) those distributions where $n_{1}$ and $n_{2}$, the two copies of the largest integer $n$, are in the same box;

(II) those distributions where $n_{1}$ and $n_{2}$ are in different boxes.

We claim that there are $L e_{n-1}^{(j-1)}$ distributions for Case (I). Indeed, if $n_{1}$ and $n_{2}$ are in the same box, it is necessarily one of the indistinguishable boxes. The remaining $j-1$ boxes plus the zero box have distributed among them the entries from $\left\{1_{1}, 1_{2}, 2_{1}, 2_{2}, \ldots,(n-1)_{1},(n-1)_{2}\right\}$, and this can be done in $L e_{n-1}^{(j-1)}$ ways. In determining the number of distributions satisfying the conditions of (II), suppose $n_{1}$ and $n_{2}$ are in different boxes. If we first distribute the elements $\left\{1_{1}, 1_{2}, 2_{1}, 2_{2}, \ldots,(n-1)_{1},(n-1)_{2}\right\}$ into the $j$ indistinguishable boxes plus the zero box (which we can do in $L e_{n-1}^{(j-1)}$ ways), then we can put $n_{1}$ into any of the $j+1$ boxes and we, independently, can put $n_{2}$ into any of the remaining $j$ boxes. The total number of distributions for Case (II) is therefore $j(j+1) L e_{n-1}^{(j-1)}$.

Combining these cases, we see that

$$
L e_{n}^{(j)}=L e_{n-1}^{(j-1)}+j(j+1) L e_{n-1}^{(j-1)} .
$$

In conclusion, we see that $L e_{n}^{(j)}$ and $P S_{n}^{(j)}$ satisfy the same initial conditions and defining recurrence relation. Therefore, for $n, j \geq 0$,

$$
L e_{n}^{(j)}=P S_{n}^{(j)} .
$$


Example 4.4. The argument in Example 4.2 easily generalizes to show that $P S_{n}^{(1)}=2^{n-1}$ for any $n \in \mathbb{N}$.

Example 4.5. Our last example gives a counting argument to show that $P S_{n}^{(n-1)}=$ $2\left(\begin{array}{c}n+1 \\ 3\end{array}\right)$; of course, we may verify this directly from the triangular recurrence relation in (3.1). In this case, we have the zero box and $n-1$ "other" non-empty, indistinguishable boxes to distribute two copies of each of the integers $1,2, \ldots, n$ according to the above rules. Since the least entry in each "other" box must be repeated in that box, we see that there is exactly one pair $\left\{i_{1}, i_{2}\right\}$ which is split into separate boxes. Keep in mind that $i=i_{1}=i_{2}$ can be put into an "other" box only if it is larger than the minimal pair put in that box; moreover, it is clear that $i \neq 1$. Hence, if one of the $i$ 's (either $i_{1}$ or $i_{2}$ ) goes into the zero box, the other $i\left(i_{2}\right.$ or $i_{1}$ ) can go into $i-1$ of the other boxes; the total count for all possible pairs in this instance is

$$
\sum_{i_{1}=2}^{n}\left(i_{1}-1\right)+\sum_{i_{2}=2}^{n}\left(i_{2}-1\right)=2 \sum_{i=2}^{n}(i-1) .
$$

Of course, both $i$ 's might avoid the zero box. In this case, there are

$$
\sum_{i=2}^{n}(i-1)(i-2)
$$

possible ways of distributing the $i$ 's in this instance. Therefore, the total number of distributions in this case is

$$
\begin{aligned}
P S_{n}^{(n-1)} & =2 \sum_{i=2}^{n}(i-1)+\sum_{i=2}^{n}(i-1)(i-2) \\
& =\sum_{i=2}^{n} i(i-1)=2 \sum_{i=2}^{n}\left(\begin{array}{c}
i \\
2
\end{array}\right)=2\left(\begin{array}{c}
n+1 \\
3
\end{array}\right) .
\end{aligned}
$$

In particular, $P S_{3}^{(2)}=2\left(\begin{array}{l}4 \\ 3\end{array}\right)=8$ and $P S_{4}^{(3)}=2\left(\begin{array}{l}5 \\ 3\end{array}\right)=20$; see Examples 4.1 and 4.3

\section{REFERENCES}

[1] M. Abramowitz and I. Stegun (editors), Handbook of Mathematical Functions with Formulas, Graphs, and Mathematical Tables, Dover Publications, New York, 1972. MR0167642 (29:4914)

[2] G. E. Andrews, W. Gawronski, and L. L. Littlejohn, Some properties of the Legendre-Stirling numbers, in preparation.

[3] A. Bruder, L. L. Littlejohn, D. Tuncer, and R. Wellman, Left-definite theory with applications to orthogonal polynomials, J. Comput. Appl. Math., to appear.

[4] L. Comtet, Advanced combinatorics: The art of finite and infinite expansions, D. Reidel Publishing Co., Dordrecht, 1974. MR0460128 (57:124)

[5] W. N. Everitt, Legendre polynomials and singular differential operators, Lecture Notes in Mathematics, Vol. 827, Springer-Verlag, Berlin-New York, 1980, 83-106. MR610812 (82m:34020)

[6] W. N. Everitt, L. L. Littlejohn, and R. Wellman, The left-definite spectral theory for the classical Hermite differential equation, J. Comput. Appl. Math., 121 (2000), 313-330. MR:1780053 (2001m:34065)

[7] W. N. Everitt, L. L. Littlejohn, and R. Wellman, Legendre polynomials, Legendre-Stirling numbers, and the left-definite spectral analysis of the Legendre differential expression, J. Comput. Appl. Math., 148 (2002), 213-238. MR1946196 (2003k:34089)

[8] W. N. Everitt, K. H. Kwon, L. L. Littlejohn, R. Wellman, and G. J. Yoon, Jacobi-Stirling numbers, Jacobi polynomials, and the left-definite analysis of the classical Jacobi differential expression, J. Comput. Appl. Math., 208 (2007), 29-56. MR2347735 (2008k:33046) 
[9] L. L. Littlejohn and R. Wellman, A general left-definite theory for certain self-adjoint operators with applications to differential equations, J. Differential Equations, 181(2) (2002), 280-339. MR1907144(2003e:47047)

[10] M. A. Naŭmark, Linear differential operators. II, Frederick Ungar Publishing Co., New York, 1968. MR0262880(41:7485)

[11] Å. Pleijel, On Legendre's polynomials, Mathematics Studies 21, North-Holland Publishing Co., Amsterdam, 1976, pp. 175-180. MR0454152 (56:12403)

[12] Å. Pleijel, On the boundary condition for the Legendre polynomials, Annales Academiæ Scientiarum Fennicæ, Series A I Mathematica, 2 (1976), 397-408. MR0492509 (58:11623)

[13] H. Weyl, Über gewöhnliche Differentialgleichungen mit Singularitäten und die zugehörigen Entwicklungen willkürlicher Funktionen, Mathematische Annalen, 68 (1910), 220-269. MR.1511560

Department of Mathematics, The Pennsylvania State University, University Park, Pennsylvania 16801

E-mail address: andrews@math.psu.edu

Department of Mathematics, Baylor University, One Bear Place \#97328, Waco, TEXas 76798-7328

E-mail address: Lance_Littlejohn@baylor.edu 